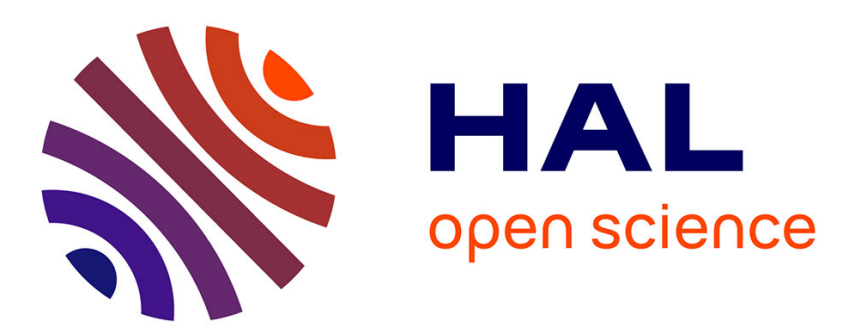

\title{
Instrumental variable scheme for closed-loop LPV model identification
}

\author{
Roland Toth, Vincent Laurain, Marion Gilson, Hugues Garnier
}

\section{To cite this version:}

Roland Toth, Vincent Laurain, Marion Gilson, Hugues Garnier. Instrumental variable scheme for closed-loop LPV model identification. Automatica, 2012, 48 (9), pp.2314-2320. 10.1016/j.automatica.2012.06.037 . hal-00765854

\section{HAL Id: hal-00765854 \\ https://hal.science/hal-00765854}

Submitted on 17 Dec 2012

HAL is a multi-disciplinary open access archive for the deposit and dissemination of scientific research documents, whether they are published or not. The documents may come from teaching and research institutions in France or abroad, or from public or private research centers.
L'archive ouverte pluridisciplinaire HAL, est destinée au dépôt et à la diffusion de documents scientifiques de niveau recherche, publiés ou non, émanant des établissements d'enseignement et de recherche français ou étrangers, des laboratoires publics ou privés. 


\title{
Instrumental variable scheme for closed-loop LPV model identification
}

\author{
Roland Tóth ${ }^{\text {a }}$, Vincent Laurain ${ }^{\mathrm{b}}$, Marion Gilson ${ }^{\mathrm{b}}$, Hugues Garnier ${ }^{\mathrm{b}}$ \\ ${ }^{a}$ Delft Center for Systems and Control, Delft University of Technology, Mekelweg 2, 2628 CD, Delft, The Netherlands \\ ${ }^{\mathrm{b}}$ Centre de Recherche en Automatique de Nancy (CRAN), Nancy-Université, CNRS, BP 70239, 54506 Vandoeuvre-les-Nancy \\ Cedex, France.
}

\begin{abstract}
Identification of real-world systems is often applied in closed loop due to stability, performance or safety constraints. However, in case of Linear Parameter-Varying (LPV) systems, closed-loop identification is not well-established despite the recent advances in prediction error approaches. Building on the available results, the paper proposes the closed-loop generalization of a recently introduced instrumental variable scheme for the identification of LPV-IO models with Box-Jenkins type of noise model structures. Estimation under closed-loop conditions with the proposed approach is analyzed from the stochastic point of view and the performance of the method is demonstrated through a representative simulation example.
\end{abstract}

Key words: Linear parameter-varying system; Identification; Refined instrumental variable; Box-Jenkins models.

\section{Introduction}

Identification of physical or chemical systems is often restricted to data gathered during closed-loop operation due to stability, performance/economical or safety constraints. As the control loop itself introduces correlation between the disturbances and the control signal, the statistically optimal (unbiased with minimal variance) estimation of the parameters of a chosen model structure w.r.t. the data-generating system is an essentially different problem than in the open-loop setting. Hence in the identification literature, especially in the linear timeinvariant (LTI) case, many approaches have been proposed to provide well-applicable solutions in this problem setting. An overview of the recent developments in the LTI case can be found in [16] and [6].

Identification of linear parameter-varying (LPV) models for systems operated in open loop has recently seen significant improvements. The case of closed-loop LPV model identification has however remained sparsely

Email addresses: rolandtoth@berkeley.edu (Roland Tóth), vincent.laurain@cran.uhp-nancy.fr (Vincent Laurain), marion.gilson@cran.uhp-nancy.fr (Marion Gilson), hugues .garnier@cran.uhp-nancy.fr (Hugues Garnier).

1 Supported by the Netherlands Organization for Scientific Research (grant no. 680-50-0927). studied (see $[5,14]$ ). The main difficulty in this system class in terms of identification is that even if the signal relations between the inputs $u$ and outputs $y$ of the system are linear, these relations are allowed to change over time as a function of a so-called scheduling variable $p: \mathbb{Z} \rightarrow \mathbb{P}$ with $\mathbb{P} \subseteq \mathbb{R}^{n_{\mathrm{p}}}$. This allows to describe a large class of nonlinear/time-varying systems in an attractive structure on which the well-established LPV controlsynthesis approaches, e.g. [11,12], can be applied. On the other hand, this varying-relation prevents the use of crucial system theoretical relations, like transfer functions and commutativity of operators, on which most LTI closed-loop identification approaches are based. As a consequence, only preliminary closed-loop methods have been proposed in the literature without being able to exploit the existing tools and knowledge available in the LTI case. In [17], an approximation based LPV extension of a predictor subspace approach (PSBID) has been introduced which is also applicable in a closedloop setting. In [3], also an approximation based LPV extension of the CLOE algorithm (see [8]) has been investigated w.r.t. LPV output-error (OE) type of models. In [1] and [4], a basic instrumental variable method has been introduced. It is a clear indicator of the immature state of this research direction that the stochastic properties of the estimation has only been analyzed in [4]. Unfortunately in [9], it has been shown recently that the formulation of the one-step IV approach, proposed in [4], does not allow to reach statistically optimal esti- 
mates due to the non-commutativity of certain filtering operations. This highlights that up to now, no method has been established which allows statistically optimal estimation under a closed-loop setting especially with general noise models like Box-Jenkins (BJ).

Based upon the available results of $[1,4]$ and using the theoretical advancements of the LPV prediction errorframework introduced in [14], we propose in this paper a closed-loop extension of a recently developed IV approach for LPV-BJ models [9]. In this setting, the noise $v_{\mathrm{o}}$ affecting the sampled output measurement $y\left(t_{k}\right)$ is assumed to have a rational spectral density which is allowed to be unrelated to the actual process dynamics of the data-generating system (general noise structure). As a first step towards the case of a $p$-dependent noise, it is also assumed that this rational spectral density does not depend on $p$. A stochastic analysis of the proposed closed-loop approach is provided, exploring the limitations and the benefits of this estimation strategy. The performance of the algorithm is also demonstrated on a representative simulation example.

\section{Problem description}

Consider the data-generating LPV system $\mathcal{S}_{\text {o given in }}$ the closed-loop setting of Fig. 1, where $\mathcal{S}_{\mathrm{o}}$ is defined as:

$$
\begin{aligned}
A_{\mathrm{o}}\left(p_{k}, q^{-1}\right) \chi_{\mathrm{o}}\left(t_{k}\right) & =B_{\mathrm{o}}\left(p_{k}, q^{-1}\right) u\left(t_{k-d}\right), \\
y\left(t_{k}\right) & =\chi_{\mathrm{o}}\left(t_{k}\right)+v_{\mathrm{o}}\left(t_{k}\right) .
\end{aligned}
$$

Here $u\left(t_{k}\right)$ is the input of the plant, $p_{k}$ is the value of the scheduling variable $p$ at sample time $t_{k}, \chi_{\mathrm{o}}$ is the noise-free output, $v_{\mathrm{o}}$ is the additive noise with bounded spectral density, $y$ is the noisy output of the system, $d$ is the delay, and $q$ is the time-shift operator, i.e. $q^{-i} u\left(t_{k}\right)=$ $u\left(t_{k-i}\right) . A_{\mathrm{o}}\left(p_{k}, q^{-1}\right)$ and $B_{\mathrm{o}}\left(p_{k}, q^{-1}\right)$ are polynomials in $q^{-1}$ of degree $n_{\mathrm{a}}$ and $n_{\mathrm{b}}$ respectively:

$$
\begin{aligned}
& A_{\mathrm{o}}\left(p_{k}, q^{-1}\right)=1+\sum_{i=1}^{n_{\mathrm{a}}} a_{i}^{\mathrm{o}}\left(p_{k}\right) q^{-i}, \\
& B_{\mathrm{o}}\left(p_{k}, q^{-1}\right)=\sum_{j=0}^{n_{\mathrm{b}}} b_{j}^{\mathrm{o}}\left(p_{k}\right) q^{-j},
\end{aligned}
$$

where the coefficients $a_{i}^{\mathrm{o}}$ and $b_{j}^{\mathrm{o}}$ are real meromorphic functions ${ }^{2}$ with static dependence on $p$, i.e. dependence only on the instantaneous value of $p$ at time $t_{k}$. It is assumed that each $a_{i}^{o}$ and $b_{j}^{\circ}$ is non-singular on $\mathbb{P}$, thus the solutions of $\mathcal{S}_{\mathrm{o}}$ are well-defined and the process part $\mathcal{G}_{\mathrm{o}}$, defined by (1a), is completely characterized by the coefficient functions $\left\{a_{i}^{\mathrm{o}}\right\}_{i=1}^{n_{\mathrm{a}}}$ and $\left\{b_{j}^{\mathrm{o}}\right\}_{j=0}^{n_{\mathrm{b}}}$. The noise $v_{\mathrm{o}}$ is assumed to be independent from $p$. Latter we will return to the more general case of $p$-dependent noise models, showing that in case of an IV-based estimation, violation of this assumption does not lead to a biased estimate.

${ }^{2} f: \mathbb{R}^{n} \rightarrow \mathbb{R}$ is a real meromorphic function if $f=g / h$ with $g, h$ analytic and $h \neq 0$.

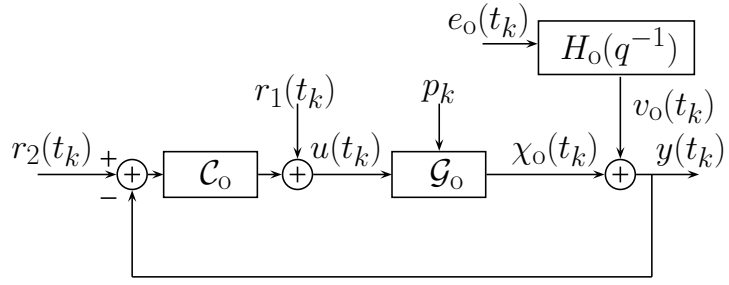

Fig. 1. Closed-loop LPV system.

If $v_{\mathrm{o}}$ has a rational spectral density, then the noise process can be represented by an autoregressive moving average (ARMA) model:

$v_{\mathrm{o}}\left(t_{k}\right)=H_{\mathrm{o}}(q) e_{\mathrm{o}}\left(t_{k}\right)=\frac{C_{\mathrm{o}}\left(q^{-1}\right)}{D_{\mathrm{o}}\left(q^{-1}\right)} e_{\mathrm{o}}\left(t_{k}\right)$,

where $C_{\mathrm{o}}\left(q^{-1}\right)$ and $D_{\mathrm{o}}\left(q^{-1}\right) \neq 0$ are monic polynomials with constant coefficients and with respective degree $n_{\mathrm{c}}$ and $n_{\mathrm{d}}$. The corresponding proper transfer function $H_{\mathrm{o}}(q)$ is assumed to be stable and to have a stable inverse. In case $C_{\mathrm{o}}\left(q^{-1}\right)=D_{\mathrm{o}}\left(q^{-1}\right)=1$, (3) defines an OE noise model, however with $C_{\mathrm{o}}\left(q^{-1}\right) \neq D_{\mathrm{o}}\left(q^{-1}\right),(3)$ is general enough to represent BJ-type of noise models. In terms of the closed-loop setting of Fig. 1, $u\left(t_{k}\right)=$ $r_{1}\left(t_{k}\right)+\mathcal{C}_{\mathrm{o}}\left(r_{2}\left(t_{k}\right)-y\left(t_{k}\right)\right)$, where $\mathcal{C}_{\mathrm{o}}$ is the operator form of the controller and $r_{1}, r_{2}$ are reference signals. Note that, as in many digital control systems, $u$ is known (the error introduced by the actuators can be modeled as an output additive ( $p$-dependent) noise due to the linearity of the system). The controller can be any LTI, nonlinear or LPV controller, under the assumptions that

- $\mathcal{C}_{\mathrm{o}}$ is a priori known;

- $\mathcal{C}_{\mathrm{o}}$ ensures BIBO stability of the closed-loop system $\mathcal{S}_{\mathrm{o}}$ for any $p \in \mathbb{P}^{\mathbb{Z}}$.

Next we introduce a model structure and parametrization for the identification of $\mathcal{S}_{\mathrm{O}}$, where, according to (1ab) and (3), the noise model and the process model are parameterized separately. The proposed LPV-BJ model, denoted in the sequel as $\mathcal{M}_{\theta}$, is defined as:

$$
\begin{aligned}
A\left(p_{k}, q^{-1}, \rho\right) \chi\left(t_{k}\right) & =B\left(p_{k}, q^{-1}, \rho\right) u\left(t_{k-d}\right), \\
D\left(q^{-1}, \eta\right) v\left(t_{k}\right) & =C\left(q^{-1}, \eta\right) e\left(t_{k}\right), \\
y\left(t_{k}\right) & =\chi\left(t_{k}\right)+v\left(t_{k}\right),
\end{aligned}
$$

with parameters $\theta=\left[\rho^{\top} \eta^{\top}\right]$ and with $u\left(t_{k}\right)=r_{1}\left(t_{k}\right)+$ $\mathcal{C}_{\mathrm{o}}\left(r_{2}\left(t_{k}\right)-y\left(t_{k}\right)\right)$. The process model part of $\mathcal{M}_{\theta}$, denoted by $\mathcal{G}_{\rho}$, is defined in terms of the LPV-IO representation (4a) where $A$ and $B$ are polynomials with order $n_{\mathrm{a}}$ and $n_{\mathrm{b}}$ respectively and with $p$-dependent coefficients $a_{i}$ and $b_{j}$ parameterized as

$a_{i}\left(p_{k}\right)=a_{i, 0}+\sum_{l=1}^{n_{\alpha}} a_{i, l} f_{l}\left(p_{k}\right), \quad i=1, \ldots, n_{\mathrm{a}}$,
$b_{j}\left(p_{k}\right)=b_{j, 0}+\sum_{l=1}^{n_{\beta}} b_{j, l} g_{l}\left(p_{k}\right), \quad j=0, \ldots, n_{\mathrm{b}}$. 
In this parametrization, $\left\{f_{l}\right\}_{l=1}^{n_{\alpha}}$ and $\left\{g_{l}\right\}_{l=1}^{n_{\beta}}$ are a priori chosen meromorphic functions of $p$ bounded on $\mathbb{P}$, with static dependence, allowing the identifiability of the model (e.g. linearly independent functions on $\mathbb{P}$ ). The associated model parameters $\rho$ are stacked columnwise: $\rho=\left[\begin{array}{llllll}\mathrm{a}_{1} & \ldots & \mathrm{a}_{n_{\mathrm{a}}} & \mathrm{b}_{0} & \ldots & \mathrm{b}_{n_{\mathrm{b}}}\end{array}\right]^{\top} \in \mathbb{R}^{n_{\rho}}$ where $\mathrm{a}_{i}=\left[\begin{array}{llll}a_{i, 0} & a_{i, 1} & \ldots & a_{i, n_{\alpha}}\end{array}\right], \mathrm{b}_{j}=\left[\begin{array}{llll}b_{j, 0} & b_{j, 1} & \ldots & b_{j, n_{\beta}}\end{array}\right]$ and $n_{\rho}=n_{\mathrm{a}}\left(n_{\alpha}+1\right)+\left(n_{\mathrm{b}}+1\right)\left(n_{\beta}+1\right)$.

The noise-model part of $\mathcal{M}_{\theta}$, denoted by $\mathcal{H}_{\eta}$, is defined in terms of (4b) where $C$ and $D$ are monic polynomials with order $n_{\mathrm{c}}$ and $n_{\mathrm{d}}$ respectively and with constant coefficients $c_{i}$ and $d_{j}$ collected as $\eta=\left[\begin{array}{llllll}c_{1} & \ldots & c_{n_{\mathrm{c}}} & d_{1} & \ldots & d_{n_{\mathrm{d}}}\end{array}\right]^{\top} \in \mathbb{R}^{n_{\eta}}$, where $n_{\eta}=$ $n_{\mathrm{c}}+n_{\mathrm{d}}$. As $(4 \mathrm{~b})$ is LTI, it can be represented by a transfer function $H(q, \eta)=C\left(q^{-1}, \eta\right) / D\left(q^{-1}, \eta\right)$. It is further assumed that $\eta \in \Theta_{\eta} \subset \mathbb{R}^{n_{\eta}}$ such that $H(z, \eta)$ is stable (analytic in the exterior of the unit circle) and has a stable inverse.

Introduce also $\mathcal{G}=\left\{\mathcal{G}_{\rho} \mid \rho \in \mathbb{R}^{n_{\rho}}\right\}$ and $\mathcal{H}=\left\{\mathcal{H}_{\eta} \mid \eta \in\right.$ $\left.\mathbb{R}^{n_{\eta}}\right\}$, as the collection of all process and noise models in the form of (4a) and (4b). Then the model set, denoted as $\mathcal{M}$, takes the form

$\mathcal{M}=\left\{\left(\mathcal{G}_{\rho}, \mathcal{H}_{\eta}\right) \mid \operatorname{col}(\rho, \eta)=\theta \in \mathbb{R}^{n_{\rho}+n_{\eta}}\right\}$,

corresponding to the set of candidate models in which we seek the model that explains data gathered from $\mathcal{S}_{\mathrm{O}}$ the best under a given identification criterion.

Denote $\mathcal{D}_{N}=\left\{y\left(t_{k}\right), u\left(t_{k}\right), p\left(t_{k}\right)\right\}_{k=1}^{N}$ a data sequence of $\mathcal{S}_{\mathrm{O}}$. In [9], it has been shown, that a one-step-ahead output predictor $\hat{y}_{\theta}\left(t_{k} \mid t_{k-1}\right)$ can be formulated w.r.t. the considered model structure (4a-c) under the commonly used assumption that noise-free observation of the sequence $\left\{p_{k}, p_{k-1}, \ldots\right\}$ is available. Recently it has been proven that using estimated moments, such a predictor can be formulated if $p$ is observed up to an additive white noise independent from $v_{\mathrm{o}}[15]$. However for the sake of simplicity, we will only consider the previous case. These results allow to formulate (in either case) the estimation of $\theta$, as the minimization of the one-step ahead prediction error: $\varepsilon_{\theta}=y\left(t_{k}\right)-\hat{y}_{\theta}\left(t_{k} \mid t_{k-1}\right)$. This minimization is formulated in terms of an identification criterion $W\left(\mathcal{D}_{N}, \theta\right)$, like the least squares criterion

$W\left(\mathcal{D}_{N}, \theta\right)=\frac{1}{N} \sum_{k=1}^{N} \varepsilon_{\theta}^{2}\left(t_{k}\right)=\frac{1}{N}\left\|\varepsilon_{\theta}\right\|_{\ell_{2}}^{2}$,

such that the parameter estimate is

$\hat{\theta}_{N}=\arg \min _{\theta \in \mathbb{R}^{n_{\rho}+n_{\eta}}} W\left(\mathcal{D}_{N}, \theta\right)$.

Based on the previous considerations, the identification problem addressed in the sequel is defined as follows:

Problem 1 Given a discrete-time LPV data-generating system $\mathcal{S}_{\mathrm{O}}$ in the closed-loop setting of Fig. 1 with a priori known stabilizing controller $\mathcal{C}_{\mathrm{O}}$. Based on the LPV-BJ model structure $\mathcal{M}_{\theta}$ defined by (4a-c) and a data set $\mathcal{D}_{N}$ collected from $\mathcal{S}_{\mathrm{O}}$, estimate the parameter vector $\theta$ as the minimization of (7) under the following assumptions:

$A 1 \mathcal{S}_{\mathrm{o}} \in \mathcal{M}$.

A2 In the parametrization (5a-b) of $A$ and $B,\left\{f_{l}\right\}_{l=1}^{n_{\alpha}}$ and $\left\{g_{l}\right\}_{l=1}^{n_{\beta}}$ are chosen such that $\mathcal{M}_{\theta}$ is identifiable.

A3 $\mathcal{D}_{N}$ is informative w.r.t. $\mathcal{M}_{\theta}$ : if $\theta_{1} \neq \theta_{2}$ then $\mathcal{M}_{\theta_{1}}$ and $\mathcal{M}_{\theta_{2}}$ lead to different prediction errors given $\mathcal{D}_{N}$. $A_{4} \mathcal{S}_{\mathrm{O}}$ is BIBO stable, i.e. for any bounded $p \in \mathbb{P}^{\mathbb{Z}}$ and $u \in \mathbb{R}^{\mathbb{Z}}$, the output of $\mathcal{S}_{\mathrm{O}}$ is bounded [14].

A5 The closed-loop system with $\mathcal{C}_{\mathrm{O}}$ is BIBO stable.

In [9], a refined instrumental variable (RIV) based approach has been introduced to solve the estimation problem of $\mathcal{M}_{\theta}$ in an open-loop setting. However, a major difference between the open-loop setting considered in [9] and the closed-loop setting of Prob. 1 is the correlation of $u$ with $e_{\mathrm{o}}$ due to the feedback loop. Therefore in the following, we aim to solve the problem of determining a suitable instrument that allows to developing the LPVRIV approach in the closed-loop case.

\section{RIV approach for closed-loop LPV systems}

In [9] it has been shown that reformulation of (4a-c) as a multiple-input single-output (MISO) LTI model allows an elegant solution of the filtering problem associated with LPV-IV approaches. This reformulation is necessary to avoid the problem of non-commutativity of parameter-varying filters and hence required to solve Prob. 1. Consequently, (4a) is rewritten as

$$
\begin{array}{r}
\underbrace{\chi\left(t_{k}\right)+\sum_{i=1}^{n_{\mathrm{a}}} a_{i, 0} \chi\left(t_{k-i}\right)}_{F\left(q^{-1}\right) \chi\left(t_{k}\right)}+\sum_{i=1}^{n_{\mathrm{a}}} \sum_{l=1}^{n_{\alpha}} a_{i, l} \underbrace{f_{l}\left(p_{k}\right) \chi\left(t_{k-i}\right)}_{\chi_{i, l}\left(t_{k}\right)} \\
=\sum_{j=0}^{n_{\mathrm{b}}} \sum_{l=0}^{n_{\beta}} b_{j, l} \underbrace{g_{l}\left(p_{k}\right) u\left(t_{k-d-j}\right.}_{u_{j, l}\left(t_{k}\right)}),
\end{array}
$$

where $F\left(q^{-1}\right)=1+\sum_{i=1}^{n_{\mathrm{a}}} a_{i, 0} q^{-i}$ and $g_{0}(\cdot) \equiv 1$. Note that in this way, the LPV-BJ model is rewritten as a MISO system with $\left(n_{\mathrm{b}}+1\right)\left(n_{\beta}+1\right)+n_{\mathrm{a}} n_{\alpha}$ inputs $\left\{\chi_{i, l}\right\}_{i=1, l=1}^{n_{\mathrm{a}}, n_{\alpha}}$ and $\left\{u_{j, l}\right\}_{j=0, l=0}^{n_{\mathrm{b}}, n_{\beta}} . F\left(q^{-1}\right)$ does not depend on $p_{k}$, thus (9) and (4b-c) have the following LTI form:

$$
\begin{aligned}
y\left(t_{k}\right)= & -\sum_{i=1}^{n_{\mathrm{a}}} \sum_{l=1}^{n_{\alpha}} \frac{a_{i, l}}{F\left(q^{-1}\right)} \chi_{i, l}\left(t_{k}\right) \\
& +\sum_{j=0}^{n_{\mathrm{b}}} \sum_{l=0}^{n_{\beta}} \frac{b_{j, l}}{F\left(q^{-1}\right)} u_{k, j}\left(t_{k}\right)+H(q) e\left(t_{k}\right) .
\end{aligned}
$$

Note that (10) is equivalent with (4a-c), but it is not a representation of the associated LPV system as it includes the lumped output variables in $\left\{\chi_{i, l}\right\}_{i=1, l=1}^{n_{\mathrm{a}}, n_{\alpha}}$. Using (10), the estimation problem of the parameters can 
be formulated as a linear regression which allows optimal solution of (7) w.r.t. LPV-BJ models. To achieve this solution, one possible way is to develop an extension of the closed-loop refined instrumental variable (RIV) approach of the LTI framework. Next we derive this extension, which, as we will see, provides an easily implementable iterative estimation scheme.

Rewrite (10) to the linear regression form:

$y\left(t_{k}\right)=\varphi^{\top}\left(t_{k}\right) \rho+\tilde{v}\left(t_{k}\right)$,

where $\tilde{v}\left(t_{k}\right)=F\left(q^{-1}, \rho\right) v\left(t_{k}\right)$ and

$$
\begin{aligned}
& \varphi\left(t_{k}\right)=\left[-y\left(t_{k-1}\right) \ldots-y\left(t_{k-n_{\mathrm{a}}}\right)-\chi_{1,1}\left(t_{k}\right) \ldots\right. \\
& \left.-\chi_{n_{\mathrm{a}}, n_{\alpha}}\left(t_{k}\right) u_{0,0}\left(t_{k}\right) \ldots u_{n_{\mathrm{b}}, n_{\beta}}\left(t_{k}\right)\right]^{\top}, \\
& \rho=\left[\begin{array}{lllllllll}
a_{1,0} & \ldots & a_{n_{\mathrm{a}}, 0} & a_{1,1} & \ldots & a_{n_{\mathrm{a}}, n_{\alpha}} & b_{0,0} & \ldots & b_{n_{\mathrm{b}}, n_{\beta}}
\end{array}\right]^{\top} .
\end{aligned}
$$

Two difficulties still remain to obtain the minimum of (7) based on (11): the regressor $\varphi\left(t_{k}\right)$ contains the unknown terms $\left\{\chi_{i, l}\left(t_{k}\right)\right\}_{i=1, l=0}^{n_{\mathrm{a}}, n_{\alpha}}$ and all of its elements are corrupted with the colored noise $v\left(t_{k}\right)$. To resolve this problem, an appropriate instrument $\zeta\left(t_{k}\right)$ can be introduced such that the estimate of $\rho$ can be given as [13]:

$$
\begin{array}{r}
\hat{\rho}_{\mathrm{XIV}}(N)=\arg \min _{\rho \in \mathbb{R}^{n_{\rho}}} \|\left[\frac{1}{N} \sum_{k=1}^{N} L(q) \zeta\left(t_{k}\right) L(q) \varphi^{\top}\left(t_{k}\right)\right] \rho \\
-\left[\frac{1}{N} \sum_{k=1}^{N} L(q) \zeta\left(t_{k}\right) L(q) y\left(t_{k}\right)\right] \|_{W}^{2}
\end{array}
$$

where $\|x\|_{W}^{2}=x^{T} W x$, with $W$ a positive definite weighting matrix and $L(q)$ is a stable pre-filter. If $G_{\mathrm{o}} \in \mathcal{G}$, the estimate (12) is consistent under the following well-known conditions ${ }^{3}$ :

C1 $\overline{\mathbb{E}}\left\{L(q) \zeta\left(t_{k}\right) L(q) \varphi^{\top}\left(t_{k}\right)\right\}$ is full column rank. C2 $\overline{\mathbb{E}}\left\{L(q) \zeta\left(t_{k}\right) L(q) \tilde{v}\left(t_{k}\right)\right\}=0$.

Moreover, the minimum variance estimator can be achieved if $[7,13,18]$ :

C3 $W=I$ and $\zeta\left(t_{k}\right)$ is chosen as the noise-free version of (11) and is therefore defined in the LPV case as:

$$
\begin{aligned}
& \zeta\left(t_{k}\right)=\left[-\stackrel{\circ}{\chi}\left(t_{k-1}\right) \ldots-\stackrel{\circ}{\chi}\left(t_{k-n_{\mathrm{a}}}\right)-\stackrel{\circ}{\chi}_{1,1}\left(t_{k}\right) \ldots\right.
\end{aligned}
$$

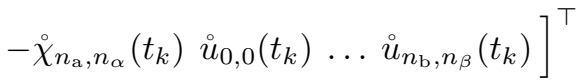

where $\dot{u}$ and $\dot{\chi}$ are the signals from the auxiliary model as presented in Fig. 2.

C4 $\mathcal{G}_{\mathrm{o}} \in \mathcal{G}$ and $n_{\rho}$ is equal to the minimal number of parameters required to represent $\mathcal{G}_{\mathrm{o}}$ with the considered model structure.

\footnotetext{
3 The notation $\overline{\mathbb{E}}\{\}=.\lim _{N \rightarrow \infty} \frac{1}{N} \sum_{t=1}^{N} \mathbb{E}\{$.$\} is adopted$
} from the prediction error framework of [10].

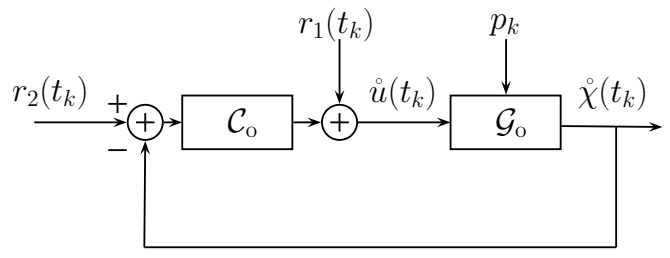

Fig. 2. Auxiliary model

C5 $L(q)$ is chosen as [9]:

$$
L(q)=\frac{D\left(q^{-1}\right)}{F\left(q^{-1}\right) C\left(q^{-1}\right)} .
$$

Full column rank of $\overline{\mathbb{E}}\left\{L(q) \zeta\left(t_{k}\right) L(q) \varphi^{\top}\left(t_{k}\right)\right\}$ is ensured by the informativity of the data set (A3). Note that conditions about informativity w.r.t. LPV-BJ model structures is in the focus of current research (see $[2,4,14]$ for results in the ARX case). To fulfill $\mathrm{C} 1$ under A3, the discussion can be found in [13]. It is also important to note that in a practical situation none of $F\left(q^{-1}, \rho\right), C\left(q^{-1}, \eta\right)$, $D\left(q^{-1}, \eta\right)$ or $\left\{a_{i, l}(\rho)\right\}_{i=1, l=0}^{n_{\mathrm{a}}, n_{\alpha}},\left\{b_{j, l}(\rho)\right\}_{j=0, l=0}^{n_{\mathrm{b}}, n_{\beta}}$ is known $a$ priori. Therefore, the RIV estimation normally involves an iterative (or relaxation) algorithm in which, at each iteration, an 'auxiliary model' is used to generate the instrumental variables (which guarantees $\mathrm{C} 2$ ), as well as the associated pre-filters. Convergence of such an iterative RIV algorithm has not been proven so far and is only empirically assumed [19].

Based on the previous considerations, the iterative scheme of the RIV algorithm can be extended to the closed-loop LPV case as described by Algorithm 1.

Based on a similar concept, the so-called simplified LPVCLRIV (LPV-CLSRIV) method, can also be developed for the estimation of LPV-OE models. This method is based on a model structure (4a-c) with $C\left(q^{-1}, \eta\right)=$ $D\left(q^{-1}, \eta\right)=1$ and consequently, Step 7 of Algorithm 1 can be skipped.

Remark 1 During the formulation of the approach it has been assumed that $v_{\mathrm{o}}$ is independent from $p$ (see (3)). However, to have a general noise model in the LPV setting it is necessary to consider p-dependence of the noise as has been pointed out in [14]. However the RIV setting, even using an OE type of noise model (like in the SRIV case), leads to unbiased estimates also in the case when the assumed noise model is incorrect (or correlated with $p$ ). The role of a more accurate noise model in this setting is to lower the variance of the resulting estimates. This explains why using a general BJ structure is important to increase the accuracy of the RIV method. However in the LPV case, allowing the noise model to be also $p$-dependent not only makes the estimation problem more difficult from the filtering point of view, but also increases significantly the number of parameters to be estimated. This results in a trade-off problem, where the simple estimation scheme proposed in this paper provides an attractive and practically applicable approach. Finding an 


\section{Algorithm 1 LPV-CLRIV \\ 1: set $\tau=0$ and let $\hat{\theta}^{(0)}=\left[\left(\hat{\rho}^{(0)}\right)^{\top}\left(\hat{\eta}^{(0)}\right)^{\top}\right]^{\top}$ be an initial parameter estimate given by the least square (LS) approach (ARX estimate of $\mathcal{M}_{\theta}$ ). \\ 2: repeat \\ 3: $\quad$ compute an estimate of $\chi\left(t_{k}\right)$ via

$$
A\left(p_{k}, q^{-1}, \hat{\rho}^{(\tau)}\right) \hat{\chi}\left(t_{k}\right)=B\left(p_{k}, q^{-1}, \hat{\rho}^{(\tau)}\right) u\left(t_{k-d}\right),
$$

where $\mathcal{G}_{\hat{\rho}(\tau)}$ is assumed to be stable (A5) and compute $\left\{\hat{\chi}_{i, l}\left(t_{k}\right)\right\}_{i=1, l=0}^{n_{\mathrm{a}, n_{\alpha}}}$ according to (9).

4: compute the estimated filter:

$$
\hat{L}\left(q^{-1}, \hat{\theta}^{(\tau)}\right)=\frac{D\left(q^{-1}, \hat{\eta}^{(\tau)}\right)}{C\left(q^{-1}, \hat{\eta}^{(\tau)}\right) F\left(q^{-1}, \hat{\rho}^{(\tau)}\right)}
$$

and the filtered signals $\left\{u_{j, l}^{\mathrm{f}}\left(t_{k}\right)\right\}_{j=0, l=0}^{n_{\mathrm{b}}, n_{\beta}}, y_{\mathrm{f}}\left(t_{k}\right)$ and $\left\{\hat{\chi}_{i, l}^{\mathrm{f}}\left(t_{k}\right)\right\}_{i=1, l=0}^{n_{\mathrm{a}}, n_{\alpha}}$.

5: build the estimated filtered regressor as:

$$
\begin{gathered}
\hat{\varphi}_{\mathrm{f}}\left(t_{k}\right)=\left[-y_{\mathrm{f}}\left(t_{k-1}\right) \ldots-y_{\mathrm{f}}\left(t_{k-n_{\mathrm{a}}}\right)-\hat{\chi}_{1,1}^{\mathrm{f}}\left(t_{k}\right)\right. \\
\left.\ldots-\hat{\chi}_{n_{\mathrm{a}}, n_{\alpha}}^{\mathrm{f}}\left(t_{k}\right) u_{0,0}^{\mathrm{f}}\left(t_{k}\right) \ldots u_{n_{\mathrm{b}}, n_{\beta}}^{\mathrm{f}}\left(t_{k}\right)\right]^{\top}
\end{gathered}
$$

and compute the filtered instrument $\hat{\zeta}_{\mathrm{f}}\left(t_{k}\right)$ by simulating $\mathcal{G}_{\hat{\rho}^{(\tau)}}$ according to Fig. 2:

$$
\begin{gathered}
\hat{\zeta}_{\mathrm{f}}\left(t_{k}\right)=\left[-\hat{\dot{\hat{\chi}}}_{\mathrm{f}}\left(t_{k-1}\right) \ldots-\hat{\hat{\chi}}_{\mathrm{f}}\left(t_{k-n_{\mathrm{a}}}\right)-\hat{\hat{\chi}}_{1,1}^{\mathrm{f}}\left(t_{k}\right)\right. \\
\left.\ldots-\hat{\hat{\chi}}_{n_{\mathrm{a}}, n_{\alpha}}^{\mathrm{f}}\left(t_{k}\right) \hat{\hat{u}}_{0,0}^{\mathrm{f}}\left(t_{k}\right) \ldots \hat{\mathrm{u}}_{n_{\mathrm{b}}, n_{\beta}}^{\mathrm{f}}\left(t_{k}\right)\right]^{\top}
\end{gathered}
$$

6: With $W=I$ and $\hat{L}\left(q^{-1}, \hat{\theta}^{(\tau)}\right)$ (according to C3C5), compute the solution of (12) via

$$
\hat{\rho}^{(\tau+1)}=\left[\frac{1}{N} \sum_{k=1}^{N} \hat{\zeta}_{\mathrm{f}}\left(t_{k}\right) \hat{\varphi}_{\mathrm{f}}^{\top}\left(t_{k}\right)\right]^{-1} \sum_{k=1}^{N} \frac{1}{N} \hat{\zeta}_{\mathrm{f}}\left(t_{k}\right) y_{\mathrm{f}}\left(t_{k}\right) .
$$

7: $\quad$ compute the estimate of the noise signal $v$ as

$$
\hat{v}\left(t_{k}\right)=y\left(t_{k}\right)-\hat{\chi}\left(t_{k}, \hat{\rho}^{(\tau)}\right) \text {. }
$$

Based on $\hat{v}$, estimation of $\hat{\eta}^{(\tau+1)}$ follows using e.g. the ARMA estimation algorithm of the MATLAB identification toolbox (an IV approach can also be used for this purpose, see [19]).

8: $\quad$ increase $\tau$ by 1

9: until $\theta^{(\tau)}$ has converged

$I V$-based estimation scheme for p-dependent BJ models, which preserves the simplicity of the proposed approach, could lead to further decrease of the variance of the estimates and hence it is in the focus of future research.

\section{Simulation Example}

In the following example we aim to demonstrating the performance of proposed closed-loop RIV scheme on a relevant simulation example. In this demonstration, we will focus on estimation under different noise conditions/models, hence the model structure of the process part is assumed to be known.

Consider the example of a mass connected to a spring and a varying damper depicted in Fig. 3. This problem is one of the typical phenomena occurring in the motion control of many mechatronic systems like in active suspension. Denote $x$ the position (in [m]) of the mass $m$ (in $[\mathrm{kg}]), \mathrm{k}_{\mathrm{s}}>0$ the stiffness of the spring and $\mathrm{c}_{\mathrm{d}}>0$ the varying damping. Introduce $F$ as the force (in $[\mathrm{N}]$ ) acting on the mass $m$. Then in continuous time (CT), the behavior of the system is defined by:

$$
\frac{\mathrm{d}}{\mathrm{d} t}\left(m \frac{\mathrm{d}}{\mathrm{d} t} x(t)\right)=F(t)-\mathrm{k}_{\mathrm{s}} x(t)-\mathrm{c}_{\mathrm{d}}(t) \frac{\mathrm{d}}{\mathrm{d} t} x(t) .
$$

By considering $F(t)$ as the input $u(t), x(t)$ as the output $y(t)$ and $p(t): \mathbb{R} \rightarrow[0,1]$ as the scheduling variable such that $c_{\mathrm{d}}(t)=\mathrm{c}_{\mathrm{d}}^{(0)}+\mathrm{c}_{\mathrm{d}}^{(1)} p(t),(14)$ can be rewritten as a CTLPV system. By using a simple backward Euler type of discretization in a zero-order-hold setting with sampling period $T_{\mathrm{d}}>0$, this LPV system can be formulated as a discrete-time (DT) LPV system:

$$
\begin{array}{r}
y\left(t_{k}\right)=\frac{T_{\mathrm{d}} \mathrm{c}_{\mathrm{d}}^{(1)} p_{k}+T_{\mathrm{d}} \mathrm{c}_{\mathrm{d}}^{(0)}+2 m}{L\left(p_{k}\right)} y\left(t_{k-1}\right)- \\
\frac{m}{L\left(p_{k}\right)} y\left(t_{k-2}\right) \\
+\frac{T_{\mathrm{d}}^{2}}{L\left(p_{k}\right)} u\left(t_{k}\right)
\end{array}
$$

where $u\left(t_{k}\right), y\left(t_{k}\right), p_{k}$ denote the sampled signals of $F(t), x(t)$, and $p(t)$ respectively and $L\left(p_{k}\right)=$ $T_{\mathrm{d}} \mathrm{c}_{\mathrm{d}}^{(1)} p_{k}+T_{\mathrm{d}} \mathrm{c}_{\mathrm{d}}^{(0)}+m+\mathrm{k}_{\mathrm{s}} T_{\mathrm{d}}^{2}$. To simplify the problem, approximate $\frac{1}{L}$ by its $1^{\text {st }}$-order Taylor approximation at the mid-point of $\mathbb{P}$, i.e. at $p_{k}=0.5$ :

$\left.\frac{1}{L(p)}\right|_{p=0.5} \approx \frac{1}{\frac{\tau_{1}}{2}+\tau_{0}}-\frac{\tau_{1}}{\left(\frac{\tau_{1}}{2}+\tau_{0}\right)^{2}}(p-0.5)$,

where $\tau_{0}=T_{\mathrm{d}} \mathrm{c}_{\mathrm{d}}^{(0)}+m+\mathrm{k}_{\mathrm{s}} T_{\mathrm{d}}^{2}$ and $\tau_{1}=T_{\mathrm{d}} \mathrm{c}_{\mathrm{d}}^{(1)}$. Then the resulting DT-LPV representation reads as

$$
\begin{aligned}
y\left(t_{k}\right)= & -\left(a_{10}+a_{11} p_{k}+a_{12} p_{k}^{2}\right) y\left(t_{k-1}\right)- \\
& \left(a_{20}+a_{21} p_{k}\right) y\left(t_{k-2}\right)+\left(b_{00}+b_{01} p_{k}\right) u\left(t_{k}\right),
\end{aligned}
$$

where $a_{11}=-\tau^{\prime} T_{\mathrm{d}} \mathrm{c}_{\mathrm{d}}^{(1)}+\tau^{\prime \prime}\left(T_{\mathrm{d}} \mathrm{c}_{\mathrm{d}}^{(0)}+2 m\right), a_{10}=$ $-\tau^{\prime}\left(T_{\mathrm{d}} \mathrm{c}_{\mathrm{d}}^{(0)}+2 m\right), a_{12}=\tau^{\prime \prime} T_{\mathrm{d}} \mathrm{c}_{\mathrm{d}}^{(1)}, a_{20}=\tau^{\prime} m$, $a_{21}=-\tau^{\prime \prime} m, \quad b_{00}=\tau^{\prime} T_{\mathrm{d}}^{2}, \quad b_{01}=-\tau^{\prime \prime} T_{\mathrm{d}}^{2}$ with $\tau^{\prime}=\frac{2}{\tau_{1}+2 \tau_{0}}+\frac{2 \tau_{1}}{\left(\tau_{1}+2 \tau_{0}\right)^{2}}$ and $\tau^{\prime \prime}=\frac{4 \tau_{1}}{\left(\tau_{1}+2 \tau_{0}\right)^{2}}$.

Consider (15) as $\mathcal{G}_{\mathrm{o}}$ in the closed loop setting of Fig. 1 with parameters $T_{\mathrm{d}}=0.05 \mathrm{~s}, m=0.01, \mathrm{k}_{\mathrm{s}}=0.85$, $\mathrm{c}_{\mathrm{d}}^{(1)}=0.5, \mathrm{c}_{\mathrm{d}}^{(0)}=0.5$. To control the motion of this system an LPV-PI controller $\mathcal{C}_{\mathrm{o}}$ has been designed:

$$
\begin{aligned}
u\left(t_{k}\right)=u\left(t_{k-1}\right)+K_{\mathrm{C}}\left(p_{k}\right)\left(w\left(t_{k}\right)-w\left(t_{k-1}\right)\right) & \\
+ & \frac{K_{\mathrm{C}}\left(p_{k}\right) T_{\mathrm{d}}}{T_{\mathrm{I}}\left(p_{k}\right)} w\left(t_{k}\right),
\end{aligned}
$$




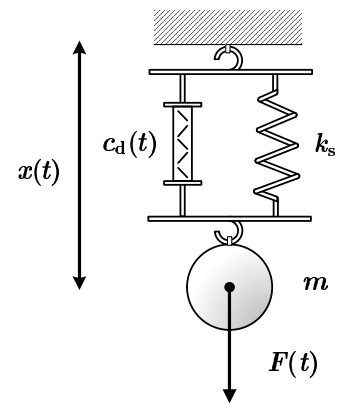

Fig. 3. Mass-spring-damper system with varying damping.

where $w\left(t_{k}\right)=r\left(t_{k}\right)-y\left(t_{k}\right), K_{\mathrm{C}}\left(p_{k}\right)=3.33+0.99 p_{k}+$ $2.06 p_{k}^{2}$ and $T_{\mathrm{I}}\left(p_{k}\right)=0.225+0.215 p_{k}-0.09 p_{k}^{2}$. Additionally, a BJ type of noise $v$ is added to the output. Consequently the data generating system can be expressed:

$\mathcal{S}_{\mathrm{o}}\left\{\begin{array}{l}A_{\mathrm{o}}\left(q, p_{k}\right)=1+a_{1}^{\mathrm{o}}\left(p_{k}\right) q^{-1}+a_{2}^{\mathrm{o}}\left(p_{k}\right) q^{-2} \\ B_{\mathrm{o}}\left(q, p_{k}\right)=b_{0}^{\mathrm{o}}\left(p_{k}\right) \\ H_{\mathrm{o}}(q)=\frac{1}{1-0.5 q^{-1}-0.2 q^{-2}}\end{array}\right.$

where $v\left(t_{k}\right)=H_{\mathrm{o}}(q) e_{\mathrm{o}}\left(t_{k}\right), e_{\mathrm{o}}\left(t_{k}\right) \in \mathcal{N}\left(0, \sigma_{\mathrm{e}}^{2}\right)$ and

$a_{1}^{\mathrm{o}}\left(p_{k}\right)=-1.135-0.1738 p_{k}+0.2538 p_{k}^{2}$,

$a_{2}^{\mathrm{O}}\left(p_{k}\right)=0.2523-0.1015 p_{k}$,

$b_{0}^{\mathrm{o}}\left(p_{k}\right)=0.0631-0.0254 p_{k}$.

In the sequel, both the scheduling signal $p$ and the input $r\left(t_{k}\right)$ are considered as white noises with uniform distributions $\mathcal{U}(0.05,0.95)$ and $\mathcal{U}(-0.5,0.5)$ respectively. Realizations of these signals with length $N=2000$ are used to generate data sets $\mathcal{D}_{N}$ of $\mathcal{S}_{\mathrm{o}}$. To demonstrate the efficiency of the presented method, the proposed approach is compared to the conventional least-squares (LPV-LS) method (see [2]) and also to the direct nonlinear minimization of the least-squares criteria (7) (NLPEM) w.r.t. the LPV-BJ model. The latter approach is computed via the LSQNONLIN algorithm of the optimization toolbox in MATLAB. Note that the NL-PEM approach provides statistically optimal results in case the model structure is optimally chosen and the nonlinear optimization succeeds to find the global minimum of (7) (depending on the initialization). Therefore to use the NL-PEM as a base-line result for assessing the performance of the IV approach, it is initialized with the true parameters and hence the provided estimates can be considered optimal.

The LPV-LS method assumes an LPV-ARX type of structure defined in this case as:

$\mathcal{M}_{\theta}^{\mathrm{LPV}-\mathrm{ARX}}\left\{\begin{array}{l}A\left(p_{k}, q^{-1}, \rho\right)=1+a_{1}\left(p_{k}\right) q^{-1}+a_{2}\left(p_{k}\right) q^{-2} \\ B\left(p_{k}, q^{-1}, \rho\right)=b_{0}\left(p_{k}\right) \\ H\left(p_{k}, q, \rho\right)=A^{\dagger}\left(p_{k}, q^{-1}, \rho\right)\end{array}\right.$

where $A^{\dagger}$ is the adjoint of $A$ and

$$
\begin{aligned}
& a_{1}\left(p_{k}\right)=a_{1,0}+a_{1,1} p_{k}+a_{1,2} p_{k}^{2}, \\
& a_{2}\left(p_{k}\right)=a_{2,0}+a_{2,1} p_{k}, \\
& b_{0}\left(p_{k}\right)=b_{0,0}+b_{0,1} p_{k} .
\end{aligned}
$$

The proposed closed-loop LPV Refined Instrumental Variable method (LPV-CLRIV) and the NL-PEM method are based on the LPV-BJ model structure:

$\mathcal{M}_{\theta}^{\mathrm{LPV}-\mathrm{BJ}}\left\{\begin{array}{l}A\left(p_{k}, q^{-1}, \rho\right)=1+a_{1}\left(p_{k}\right) q^{-1}+a_{2}\left(p_{k}\right) q^{-2} \\ B\left(p_{k}, q^{-1}, \rho\right)=b_{0}\left(p_{k}\right) \\ H\left(p_{k}, q, \eta\right)=\frac{1}{1+d_{1} q^{-1}+d_{2} q^{-2}}\end{array}\right.$

with $a_{1}\left(p_{k}\right), a_{2}\left(p_{k}\right), b_{0}\left(p_{k}\right), b_{1}\left(p_{k}\right)$ as given in (21a-c), and hence represent the situation $\mathcal{S}_{\mathrm{o}} \in \mathcal{M}$.

The robustness of the proposed and existing algorithms are investigated with respect to different signal-to-noise ratios: $\mathrm{SNR}=10 \log \frac{P_{\dot{\chi}_{0}}}{P_{v_{0}}}$, where $P_{\chi_{\mathrm{o}}}$ and $P_{v_{\mathrm{o}}}$ are the average power of the signals $\chi_{\mathrm{o}}$ and $v_{\mathrm{o}}$ respectively. To provide representative results, a Monte Carlo simulation of $N_{\mathrm{MC}}=200$ runs with new noise realizations is accomplished at SNR levels: $18 \mathrm{~dB}, 13 \mathrm{~dB}$ and $8 \mathrm{~dB}$.

Table 1 shows the norm of the bias $(\mathrm{BN})\left\|\rho_{o}-\overline{\mathbb{E}}(\hat{\rho})\right\|_{\ell_{2}}$ and variance norm $(\mathrm{VN})\|\overline{\mathbb{E}}(\hat{\rho}-\overline{\mathbb{E}}(\hat{\rho}))\|_{\ell_{2}}$ of the estimated parameter vector w.r.t. the process part, where $\overline{\mathbb{E}}$ is the mean operator over the Monte Carlo simulation. The table also displays the mean number of iterations (Nit) the algorithms needed to converge to the estimated parameter vector. Table 1 demonstrates that the LPV-CLRIV method is unbiased according to the theoretical results, while the LPV-LS method exhibits a considerable bias. For SNR down to 8dB, the LPV-CLRIV achieves a lower variance for $\hat{\rho}$ than the LPV-LS method. Moreover, the achieved variance is close to result of the statistically optimal NL-PEM estimates.

Table 1

Bias and variance results using the full model structure $\mathcal{M}_{\theta}^{\mathrm{LPV}-\mathrm{BJ}}$.

\begin{tabular}{|c||c||c|c|c|}
\hline Method & & $18 \mathrm{~dB}$ & $13 \mathrm{~dB}$ & $8 \mathrm{~dB}$ \\
\hline LPV-LS & BN & 0.0849 & 0.2158 & 0.4134 \\
\cline { 2 - 5 } & VN & 0.0752 & 0.1232 & 0.1876 \\
\hline \hline \multirow{2}{*}{ NL-PEM } & BN & $40 \mathrm{e}-04$ & $71 \mathrm{e}-04$ & 0.0138 \\
\cline { 2 - 5 } & VN & 0.0464 & 0.0819 & 0.1393 \\
\hline \hline \multirow{2}{*}{ LPV } & BN & $39 \mathrm{e}-04$ & $62 \mathrm{e}-04$ & 0.0103 \\
\cline { 2 - 5 } CLRIV & VN & 0.0505 & 0.0922 & 0.1638 \\
\cline { 2 - 5 } & Nit & 9 & 12 & 12 \\
\hline
\end{tabular}

To show the advantage of the proposed IV method w.r.t. NL-PEM approach, consider a more realistic scenario when the model structure of the noise is unknown. Thus, identification of $\mathcal{S}_{\mathrm{O}}$ is considered with the following LPVOE model structure (corresponding to $\mathcal{G}_{\mathrm{o}} \in \mathcal{G}, \mathcal{H}_{\mathrm{o}} \notin \mathcal{H}$ ):

$\mathcal{M}_{\theta}^{\mathrm{LPV}-\mathrm{OE}}\left\{\begin{array}{l}A\left(p_{k}, q^{-1}, \rho\right)=1+a_{1}\left(p_{k}\right) q^{-1}+a_{2}\left(p_{k}\right) q^{-2} \\ B\left(p_{k}, q^{-1}, \rho\right)=b_{0}\left(p_{k}\right) q^{-1}+b_{1}\left(p_{k}\right) q^{-2} \\ H\left(p_{k}, q, \eta\right)=1\end{array}\right.$ 
This model is identified using the proposed simplified closed-loop RIV approach (LPV-CLSRIV) and the NLPEM method. The results are presented in Table 2. It appears that the NL-PEM is affected by the incorrect structure of the noise model both in terms of bias and variance, while the proposed LPV-CLSRIV method only suffers from a mild variance increase (remaining close to the LPV-LS variance) but remains unbiased. Consequently, this representative example suggests that the proposed method is robust to the modeling error on the noise and displays a better bias/variance trade-off.

Table 2

Bias and variance results using the inaccurate model structure $\mathcal{M}_{\theta}^{\mathrm{LPV}-O \mathrm{OE}}$.

\begin{tabular}{|c||c||c|c|c|}
\hline Method & & $18 \mathrm{~dB}$ & $13 \mathrm{~dB}$ & $8 \mathrm{~dB}$ \\
\hline LPV-LS & BN & 0.0849 & 0.2158 & 0.4134 \\
\cline { 2 - 5 } & VN & 0.0752 & 0.1232 & 0.1876 \\
\hline \hline \multirow{2}{*}{ NL-PEM } & BN & 0.010 & 0.027 & 0.072 \\
\cline { 2 - 5 } & VN & 0.0621 & 0.1069 & 0.1742 \\
\hline \hline LPV & BN & $34 \mathrm{e}-04$ & $59 \mathrm{e}-04$ & 0.0134 \\
\cline { 2 - 5 } CLSRIV & VN & 0.0612 & 0.1085 & 0.2124 \\
\cline { 2 - 5 } & Nit & 13 & 15 & 18 \\
\hline
\end{tabular}

\section{Conclusion}

In this paper, a closed-loop LPV-RIV approach is introduced to provide an efficient solution for the closed-loop identification of LPV systems with Box-Jenkins type of noise models. The approach is formulated with the assumption of prior knowledge of the controller but without any restriction on its structure. It is shown that under given conditions the proposed method provides consistent estimates. The performance of the approach is demonstrated on a representative example pointing out that its particular advantage is being robust to noise and modeling errors.

\section{References}

[1] H. Abbas and H. Werner. An instrumental variable technique for open-loop and closed-loop identification of input-output LPV models. In Proc. of the European Control Conf., pages 2646-2651, Budapest, Hungary, 2009.

[2] B. Bamieh and L. Giarré. Identification of linear parameter varying models. Int. Journal of Robust and Nonlinear Control, 12:841-853, 2002.

[3] S. Boonto and H. Werner. Closed-loop system identification of LPV input-output models - application to an arm-driven pendulum. In Proc. of the 47th IEEE Conf. on Decision and Control, pages 2606-2611, Cancun, Mexico, Dec. 2008.

[4] M. Butcher, A. Karimi, and R. Longchamp. On the consistency of certain identification methods for linear parameter varying systems. In Proc. of the 17th IFAC World Congress, pages 4018-4023, Seoul, Korea, July 2008.

[5] F. Casella and M. Lovera. LPV/LFT modelling and identification: overview, synergies and a case study. In IEEE International Symposium on Computer-Aided Control System Design, pages 852-857, San Antonio, Texas, USA, Sept. 2008.
[6] U. Forssell and L. Ljung. Closed-loop identification revisited. Automatica, 35(7):1215-1241, 1999.

[7] M. Gilson and P. M. J. Van den Hof. Instrumental variable methods for closed-loop system identification. Automatica, 41(2):241-249, 2005.

[8] I. D. Landau and A. Karimi. Recursive algorithms for identification in closed loop: A unified approach and evaluation. Automatica, 33(8):1499-1523, 1997.

[9] V. Laurain, M. Gilson, R. Tóth, and H. Garnier. Refined instrumental variable methods for identification of LPV BoxJenkins models. Automatica, 46(6):959-967, 2010.

[10] L. Ljung. System Identification, theory for the user. Prentice Hall, 1999.

[11] A. Packard. Gain scheduling via linear fractional transformations. Systems \& Control Letters, 22(2):79-92, 1994.

[12] C. W. Scherer. Mixed $\mathcal{H}_{2} / \mathcal{H}_{\infty}$ control for time-varying and linear parametrically-varying systems. Int. Journal of Robust and Nonlinear Control, 6(9-10):929-952, 1996.

[13] T. Söderström and P. Stoica. Instrumental Variable Methods for System Identification. Springer-Verlag, New York, 1983.

[14] R. Tóth. Modeling and Identification of Linear ParameterVarying Systems. Lecture Notes in Control and Information Sciences, Vol. 403. Springer-Germany, 2010.

[15] R. Tóth, E. Bitar, P. S. C. Heuberger, P. M. J. Van den Hof, and K. Poolla. A prediction-error identification framework for linear parameter-varying systems. Submitted to Automatica, 2011.

[16] P. M. J. Van den Hof. Closed-loop issues in system identification. Annual reviews in control, 22:173-186, 1998.

[17] J. W. van Wingerden and M. Verhaegen. Subspace identification of bilinear and LPV systems for open- and closed-loop data. Automatica, 45(2):372-381, 2009.

[18] P. C. Young. Recursive Estimation and Time-Series Analysis. Springer-Verlag, Berlin, 1984.

[19] P. C. Young. The refined instrumental variable method: Unified estimation of discrete and continuous-time transfer function models. Journal Européen des Systèmes Automatisés, 42:149-179, 2008. 